\title{
Dental implications of bisphosphonate therapy in osteogenesis imperfecta.
}

SADJ October 2017, Vol 72 no 9424 - p428

M Chetty', T Roberts², LXG Stephen ${ }^{3}$, P Beighton ${ }^{4}$

\begin{abstract}
Bisphosphonate therapy, which is effective in reducing the rate of fracturing, represents a significant advance in the medical management of Osteogenesis imperfecta (OI). When administered to affected persons, bisphosphonate therapy is relevant in their dental and craniofacial management. A particular concern is bisphosphonateinduced osteonecrosis of the jaws, a rare but potentially devastating problem.
\end{abstract}

Osteogenesis imperfecta type III (OI III) is relatively common amongst the indigenous Black African population of South Africa. With the co-operation of medical colleagues 64 Black South African individuals with OI III, between the ages of three months and 18 years, were identified and were dentally assessed. Fifty-five of these individuals had received or were receiving bisphosphonate therapy. This impacted on dental treatment planning and delivery since there is arisk of the complication of bisphosphonate induced osteonecrosis of the jaws.

There is a paucity of information regarding the dental management of persons with $\mathrm{Ol}$ who are receiving bisphosphonate therapy. In particular, orthodontists and maxillofacial surgeons have expressed concern regarding the advisability of orthodontic extractions, tooth

1. M Chetty: $B S C, B C h D, M C h D, P h D$. Faculty of Dentistry, University of the Western Cape. University of the Western Cape/ University of Cape Town Combined Dental Genetics Clinic, Red Cross Childrens Hospital, Cape Town, South Africa.

2. T Roberts: BChD, MChD. Faculty of Dentistry, University of the Western Cape. University of the Western Cape/ University of Cape Town Combined Dental Genetics Clinic, Red Cross Childrens Hospital, Cape Town, South Africa.

3. LXG Stephen: BChD,PhD. Faculty of Dentistry, University of the Western Cape. University of the Western Cape/ University of Cape Town Combined Dental Genetics Clinic, Red Cross Childrens Hospital, Cape Town, South Africa.

4. P Beighton: $P h D, M D, F R C P$. Division of Human Genetics, Faculty of Health Sciences, University of Cape Town. University of the Western Cape/ University of Cape Town Combined Dental Genetics Clinic, Red Cross Childrens Hospital, Cape Town, South Africa.

\section{Corresponding author}

\section{Chetty:}

Tel: 0219373112

E-mail: drmchetty@mweb.co.za

\section{ACRONYMS \\ AR: autosomal recessive \\ Ol: Osteogenesis imperfecta \\ OI III: steogenesis imperfecta type III \\ ONJ: Osteonecrosis of the jaw}

movement when faced with the potential for developing osteonecrosis of the jaws.

In view of these uncertainties, the dental implications of bisphosphonate therapy have been reviewed and discussed in this article.

\section{INTRODUCTION}

Bisphosphonates are synthetic analogs of pyrophosphates, which, being deposited on the bone surface, inhibit bone resorption. They are then ingested by osteoclasts with consequent apoptosis of these cells. Bisphosphonates also demonstrate anti-angiogenic activity by inhibiting vascular endothelial growth factor and the formation of new blood vessels.1 Bisphosphonate therapy is used for several conditions notably osteoporosis, Paget's disease, metastatic bone malignancies and Osteogenesis imperfecta (OI). Although impressive radiological and clinical improvements following bisphosphonate therapy have been observed and are well documented in persons with different forms of $\mathrm{Ol}$, nevertheless bisphosphonateinduced osteonecrosis of the jaw (ONJ) remains a rare but potentially devastating problem.

In South Africa, there is a need to record clinical experiences and to devise and develop a clinical management protocol and guidelines for optimizing dental therapy in persons affected with OI who have received bisphosphonate therapy.

\section{PATIENTS AND METHODS}

All investigations were undertaken in complete accordance with the Declaration of Helsinki, the Hippocratic Oath and the Singapore Statement on Research Integrity. Formal ethical approval (HREC reference number: 203/2013) was obtained from the Ethics Committee, University of Cape Town. Informed signed consent was obtained from parents, or from guardians of affected children where appropriate. 
Severe autosomal recessive (AR) Osteogenesis imperfecta type III (OI III) is relatively common amongst the indigenous Black African population of South Africa. With the co-operation of medical colleagues, 64 Black African individuals with OI III in South Africa, between the ages of 3 months and 19 years, were identified and dentally assessed. Fifty-five of these individuals had received or were receiving bisphosphonate therapy and a detailed account of this aspect of management of OI III on 26 of these persons has recently been documented. ${ }^{2}$

An extensive appraisal of the literature in terms of dental management in conjunction with bisphosphonate therapy in OI has been undertaken and the findings and conclusions form the subject of this paper.

\section{DISCUSSION}

Pamidronate is a bisphosphonate frequently used in children. A study which included 26 Black African patients with OI III in South Africa who had received Pamidronate therapy, concluded that this treatment was well tolerated. The affected persons ranged in age from 1.5 years to 24 years and the majority reported a reduction in symptoms and an increased sense of well-being. ${ }^{2}$ No reported instances of Pamidronate being associated with osteonecrosis of the jaws in Ol could be found in the literature. In circumstances where affected persons did not respond optimally to Pamidronate, Zoledronic acid has been used. It may be relevant that Zoledronic acid has frequently been used in South African patients. Although concern exists that this form of therapy has been associated with aseptic necrosis of the jaw in adult persons, none of the South African patients presented with any symptoms of ONJ.2

\section{Bisphosphonate Therapy and Dentistry}

The jaws are susceptible to osteonecrosis due to several anatomical and physiological factors. Bisphosphonates tend to accumulate in the bones of the jaws due to the high vascularity and turnover rate. The forces of mastication and consequent tension on the periodontal ligament ensure a high turnover rate of alveolar bone and the thin oral mucosa can easily be traumatized during dental procedures, allowing oral microbes to track into the mucoperiosteal region of the jaws. ${ }^{3}$ The pathophysiology of $\mathrm{ONJ}$ is multifactorial, involving factors such as marked suppression of angiogenesis, altered functioning of oral mucosal cells, modification of the oral microbial flora, an anti-inflammatory effect and a genetic predisposition. ${ }^{4}$ It is relevant that bisphosphonate uptake results in decreased remodeling of the alveolar bone and a sclerotic lamina dura. ${ }^{5}$

A search of the literature suggests that various clinical settings in dentistry may potentially be involved if an individual has a history of or is currently receiving bisphosphonate therapy. ${ }^{5-31}$ Several case reports and cohort studies have linked bisphosphonate therapy and osteonecrosis of the jaw (ONJ) in adults in different disorders. ${ }^{1,3,6-8}$ Reports of three hundred and sixtyeight cases of ONJ have been published in a systematic review, ${ }^{9}$ the majority occurring in elderly persons with malignancies. However, to date, there have been no published data of the frequency of $\mathrm{ONJ}$ in children. The only dental report which was found pertaining to children, referred to a 1.67-year delay in tooth eruption in 33 persons with $\mathrm{OI}$ who received bisphosphonate therapy. ${ }^{10}$

A retrospective Swedish ${ }^{11}$ survey ${ }^{11}$, identified a large series of persons with various forms of $\mathrm{Ol}$, between the ages of 2 months and 20 years, who had received or were on bisphosphonate therapy, many of whom had had dental surgical procedures. No evidence of ONJ were identified in any of these patients at their eight-year follow-up.

There were no reports or history of ONJ subsequent to dental therapy amongst the 55 persons in the current South African series who were affected with OI III and who had received or were receiving bisphosphonate therapy.

\section{Dental Implants}

Persons with $\mathrm{Ol}$ have an increased risk of early loss of teeth and, hence, rehabilitation using dental implants may be a management option. Although bone graft surgery and dental implants are considered potential risk factors for the development of ONJ in individuals receiving bisphosphonate therapy, studies of this possibility have reported no incidences of ONJ in persons with Ol. ${ }^{12}$

\section{Periodontics}

The potential beneficial effects of bisphosphonates on periodontal disease have been explored, for the density of alveolar bone is increased, a favourable outcome, but, paradoxically, there an increased risk of $\mathrm{ONJ} .{ }^{5,13}$ A reported incidence of the development of osteochemonecrosis in a patient who received non-surgical periodontal treatment highlights the importance of practitioners being cognizant of this possible debilitating consequence, even when nonsurgical therapy is performed. Since $\mathrm{ONJ}$ is difficult to predict and prevent the avoidance of surgery has been advised. ${ }^{14}$

In Ol affected individuals, the lamina dura is absent, suggesting decreased mineralization of the alveolar bone (Figure 1). This factor places these persons at risk for the development of periodontal disease and consequent alveolar bone loss. The presence of periodontal disease may necessitate invasive periodontal procedures or dental extraction, which will increase the risk of ONJ.

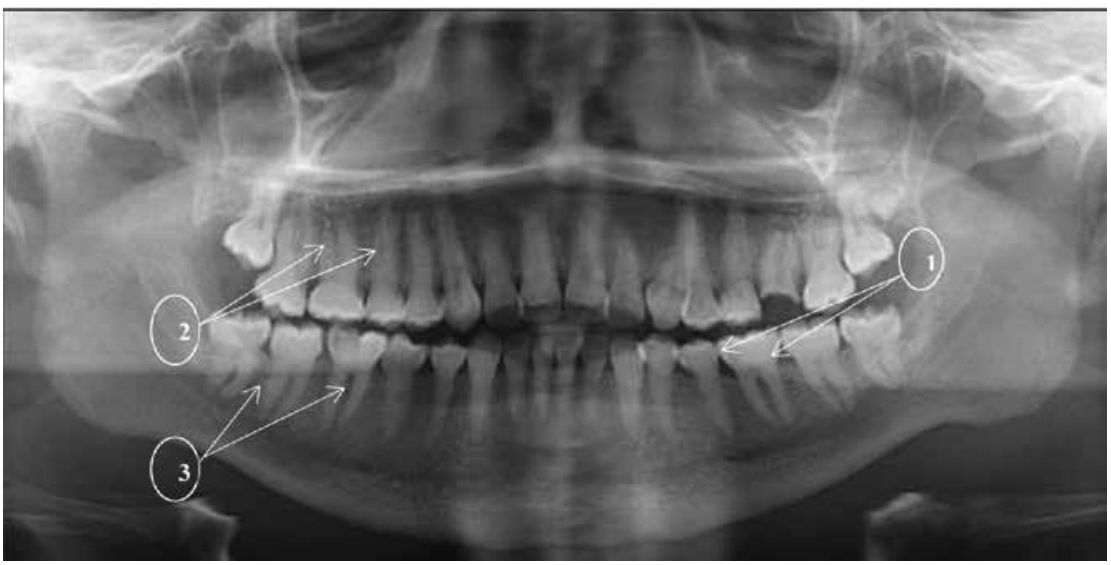

Figure 1: Panorex radiograph of a 19 year old boy with Ol. The lamina dura is absent and several radiographic features of Dentinogenesis Imperfecta are evident in all his teeth.

There is cervical constriction of the molars (1). The pulp chambers are partially or completely obliterated in almost all of his teeth (2). The roots are thin and short (3). 
For this reason, impeccable oral hygiene practices are encouraged in persons with $\mathrm{Ol}$ who are receiving bisphosphonate therapy.

Despite a vigorous literature search, no published reports were found which documented persons with $\mathrm{Ol}$ and $\mathrm{ONJ}$ consequent to periodontal therapy.

\section{Orthodontics}

Bisphosphonates are effective in the medical management of children with OI. No instances of ONJ have been reported and the extraction of teeth is not contraindicated in these children.15,16 The current authors suggest that a young age may be a protective factor.

Increasingly, adults with Ol with bisphosphonate exposure are now seeking orthodontic care. Nevertheless, a literature search has not revealed any case reports of ONJ developing during orthodontic management. Bisphosphonates may compromise orthodontic treatment in that tooth movement involves bone resorption and deposition. It has been shown that bisphosphonates can reduce the rate of orthodontic tooth movement due to their inhibition of bone resorption by osteoclast apoptosis and reduced bone vasculature. Inhibition of orthodontic tooth movement has been documented in four cases with a history of bisphosphonate exposure.17,18 No studies have specifically implicated orthodontic management as a factor in increased ONJ risk, but there is evidence that prolonged orthodontic treatment in patients suffering $\mathrm{Ol}$ may intensify the potential for ONJ. ${ }^{19}$

\section{Endodontics}

In persons with $\mathrm{OI}$ and a history of bisphosphonate therapy, endodontic treatment is preferred over extraction in order to minimize the risk of ONJ. ${ }^{20}$

\section{Oral Surgery}

A report documents several middle aged to elderly medically compromised patients on bisphosphonate therapy who presented with unusual non-healing extraction wounds. In this clinical setting, namely, bisphosphonate therapy in medically compromised individuals, it is imperative that a detailed medical history is obtained, in particular information on bisphosphonate usage and that proper procedure is followed in order to avoid the onset of ONJ. ${ }^{21}$ Dental extractions and surgical procedures need to be as atraumatic as possible and good oral hygiene is crucial in order to ensure optimal healing. These constraints are especially relevant in a bisphosphonate induced environment of reduced blood supply, sclerotic bone and reduced bone turnover. ${ }^{5,22}$

\section{Cranial Base Abnormalities}

Pathology of the cranio-cervical and base of skull region in Ol can be divided into platybasia, basilar invagination and basilar impression and it is suggested that these complications can occur separately or concurrently. ${ }^{23-} 25$ Cranial base anomalies impact on dental therapy and caution is warranted when a patient's head is manipulated in order to avoid atlanto-axial subluxation and spinal cord compression. In cephalometric analyses of dentofacial morphology, details on the cranial base constitute important reference points. This situation necessitates awareness by the dental clinician in order to enable the distinction between pathology and normal developmental patterns.
A research report documented the natural progression of cranial base anomalies in 150 persons with Ol, aged between 0 - 39 years and recorded that $37 \%$ had abnormalities. ${ }^{23}$ The authors subsequently recommended a radiological surveillance strategy with regular follow-up. In this cohort of patients, the number of individuals on bisphosphonate therapy was low; hence, a further study was undertaken in order to resolve the issue of the effect of bisphosphonates on the development of cranial base abnormalities. ${ }^{24}$ The conclusion was that although the early initiation of bisphosphonate treatment may defer the development of cranial base pathology, abnormalities may still arise despite this therapy.

\section{Antibiotic Prophylaxis}

A survey of dental specialists and dentists in the UK revealed that their approaches to the management of paediatric patients on bisphosphonates ranged from no precautions against $\mathrm{ONJ}$, to antibiotic prophylaxis. It also highlighted the fact that $\mathrm{OI}$ centres in the UK frequently received calls from dental practitioners requesting management advice for this group of individuals and it is evident that guidance in this regard is necessary. ${ }^{26}$

The question of antibiotic prophylaxis during dental intervention arose in the context of South African patients with OI III had orthopedic roots. There is a paucity of published information pertaining to this situation. A few reports have suggested that antibiotic prophylaxis for ONJ is not required when dental treatment is undertaken. ${ }^{27}$ The instance of a boy aged 10 years with Ol who had dental surgery with no administration of antibiotics and without the suspension of bisphosphonate therapy has been reported. ${ }^{28}$ Eighteen months later at his follow-up appointment, he showed no signs of ONJ.

\section{Reported Dental Management Protocol}

Currently, no established protocols or guidelines have been formulated for the dental management of persons with $\mathrm{Ol}$ who are on bisphosphonate therapy. The perception that dental extractions and other oral surgical procedures in children should be carried out only when necessary is based upon experience with adult individuals receiving bisphosphonate therapy. Delaying or avoiding the extraction of severely carious teeth may result in infection and chronic pain which can have serious consequences. Similarly, a delay or failure to manage an evolving malocclusion may deny paediatric patients a functional occlusion.

A suggested management approach is to discontinue bisphosphonate therapy 8 - 15 days prior to simple procedures such as a dental extraction and four months prior to invasive surgery such as an osteotomy. In both circumstances, antibacterial prophylaxis is recommended. ${ }^{16,29}$ It has also been documented that although bisphosphonate therapy withdrawal may not interfere with the bisphosphonate previously assimilated into the bone, withdrawal of therapy may expedite the healing process of the injured tissues by averting the antiangiogenic effect of bisphosphonates. ${ }^{30}$

Referring to patients in whom bisphosphonate therapy has already been initiated, the American Association 
of Oral and Maxillofacial Surgeons ${ }^{29}$ and the Japanese 'Allied Task Force Committee of BisphosphonateRelated Osteonecrosis of the Jaw' suggest that dental procedures be performed prior to the bisphosphonate dose reaching a high level. ${ }^{31}$ It is recommended that, prior to the commencement and during the administration of bisphosphonate therapy in this group of patients, they be referred to a dental clinician for examination, for the identification of any risk factors for ONJ, and for the development of an appropriate treatment plan and frequent follow-up. ${ }^{31}$

In the South African situation, the authors advocate that, in order to obtain baseline information of the relation between dental and craniofacial manifestations and bisphosphonate therapy, cephalometric radiographs of affected persons who have not received bisphosphonate infusions should be compared with those of Ol affected persons who have received bisphosphonate therapy. In this way, the effect of bisphosphonate therapy on the dental and craniofacial structures may be identified.

\section{CONCLUDING COMMENTS}

Most reported cases of ONJ related to bisphosphonate therapy have been in patients older than 60 years and often associated with malignancies. Osteonecrosis of the jaws has also been seen in children and adolescents with a history of malignancies who were treated with bisphosphonates. $^{8}$

Several reports have suggested that there is little risk of ONJ in paediatric Ol dental patients receiving bisphosphonate therapy. ${ }^{15,16,22}$ The reasons for this reduced risk are unclear.

When dental treatment is vital in this group of children on bisphosphonate therapy, communication with the child's medical team is essential. Patients and their parents or caregivers should be informed of the importance of maintaining good oral hygiene and having regular dental evaluations in order to prevent dental disease.

It should also be considered that as bisphosphonates are retained in bone for many years, any dental intervention in this period may, in theory, result in ONJ.

The Canadian Association of Oral and Maxillofacial Surgeons established a multidisciplinary task force that reviewed all relevant research and current literature related to ONJ.7 These authors concluded that although ONJ was identified as a risk factor in oncology patients receiving high dose intravenous bisphosphonates, low dose bisphosphonate use in patients, especially children, with $\mathrm{Ol}$, did not pose a risk for the development of ONJ and no causal link was established. ${ }^{8}$

A review of the literature suggests that the risk of bisphosphonate-associated $\mathrm{ONJ}$ in persons with $\mathrm{OI}$ is considered to be extremely low and that persons requiring dental management should not be deprived of treatment. Prior to any dental procedures, dental practitioners should inform patients of the potential risk of $\mathrm{ONJ}$ and thereby receive informed consent. Follow-up appointments are obligatory so that healing is closely monitored. If the patient develops symptoms of $\mathrm{ONJ}$, referral for secondary care is mandatory.

\section{SUGGESTIONS FOR FUTURE RESEARCH IN SOUTH AFRICA}

A prevalence study and the frequency of persons with $\mathrm{Ol}$ receiving bisphosphonate treatment who have developed ONJ consequent to dental intervention in South African clinics is necessary. Postoperative follow-up of persons with $\mathrm{Ol}$ who have received bisphosphonate therapy is important as well as documentation of the antibiotic regimen and the type of bisphosphonate used.

Osteonecrosis of the jaws is a serious clinical problem and, therefore, in order to obtain data in the South African context and to create a greater awareness of this potentially devastating concern, longitudinal studies, such as those mentioned, are necessary.

\section{References}

1. Varun BR, Sivakumar TT, Nair BJ, et al. Bisphosphonate induced osteonecrosis of the jaw in breast cancer patients: A systematic review. J Oral and Maxillofac Path. 2012; 16(2):210-213.

2. Henderson $\mathrm{BD}$, Isaac $\mathrm{N}$, Mabele $\mathrm{O}$, et al. Pamidronate treatment for osteogenesis imperfecta in black South Africans. SAMJ. 2016; 106(6):S47-S49.

3. Marx RE, Sawatari Y, Fortin M, et al. Bisphosphonate- induced exposed bone (osteonecrosis/osteopetrosis) of the jaws: Risk factors, recognition, prevention and treatment. J Oral and Maxillofac Surg. 2005; 63:1567-75.

4. Allen MR, Burr DB. The pathogenesis of bisphosphonaterelated osteonecrosis of the jaw: so many hypotheses, so few data. J Oral Maxillofac Surg. 2009; 67:61-70.

5. Borromeo GL, Tsao CE, Darby IB, et al. A review of the clinical implications of bisphosphonates in dentistry. Aust Dent J. 2011; 56: 2-9.

6. Marx RE. Pamidronate (Aredia) and Zoledronate (Zometa)induced avascular necrosis of the jaws: a growing epidemic. J Oral Maxillofac Surg. 2003; 61:1115-7.

7. Khan AA, Sandor GKB, Dore E. et al. Canadian consensus practice guidelines for bisphosphonate- associated osteonecrosis of the jaw. J Rheumatol. 2008; 35:1391-7.

8. Barasch A, Cunha-Cruz J, Curro FA. et al. Risk factors for osteonecrosis of the jaws: a case-control study from the CONDOR Dental PBRN. J Dent Res. 2011; 90(4):439-44.

9. Woo SB, Hellstein JW, Kalmar JR. Systematic review: bisphosphonates and osteonecrosis of the jaw. Ann Intern Med. 2006; 144:753-61.

10. Kamoun-Goldrat A, Ginisty D, Le Merrer M. Effects of bisphosphonates on tooth eruption in children with osteogenesis imperfecta. Eur J Oral Sci. 2008; 116:195-8.

11. Malmgren B, Astrom E, Soderhall S. No osteonecrosis in jaws of young patients with osteogenesis imperfecta treated with bisphosphonates. J Oral Pathol Med. 2008; 37(4):196-200.

12. Thirunavukarasu A, Pinto HG, Seymour KG. Bisphosphonate and implant dentistry- is it safe? Prim Dent J. 2015; 4(3):30-33.

13. Ficarra $G$, Beninati $F$, Rubino I. et al. Osteonecrosis of the jaws in periodontal patients with a history of bisphosphonate treatment. J Clin Periodontol. 2005; 32:1123-8.

14. Braun E, lacono VJ. Bisphosphonates: case report of nonsurgical periodontal therapy and osteochemonecrosis. Int $\mathrm{J}$ Periodontics Restorative Dent. 2006; 26(4):315-9.

15. Brown JJ, Ramalingam L, Zacharin MR. Bisphosphonateassociated osteonecrosis of the jaw: does it occur in children? Clin Endocrinol. 2008; 68: 863-7.

16. Schwartz S, Joseph C, lera D. et al. Bisphosphonates, osteonecrosis, osteogenesis imperfecta and dental extractions: a case series. J Can Dent Ass. 2008; 74(6):537-42.

17. Rinchuse D, Sosovicka M, Robison J. et al. Orthodontic treatment of patients using bisphosphonates: a report of two cases. Am J Orthod Dentofacial Orthop. 2007; 131:321-6.

18. Goss AN. Bisphosphonates and orthodontics. Aust Orthod J. 2008; 24:56-7.

19. Ghoneima AA, Allam ES, Zunt SL. et al. Bisphosphonates 
